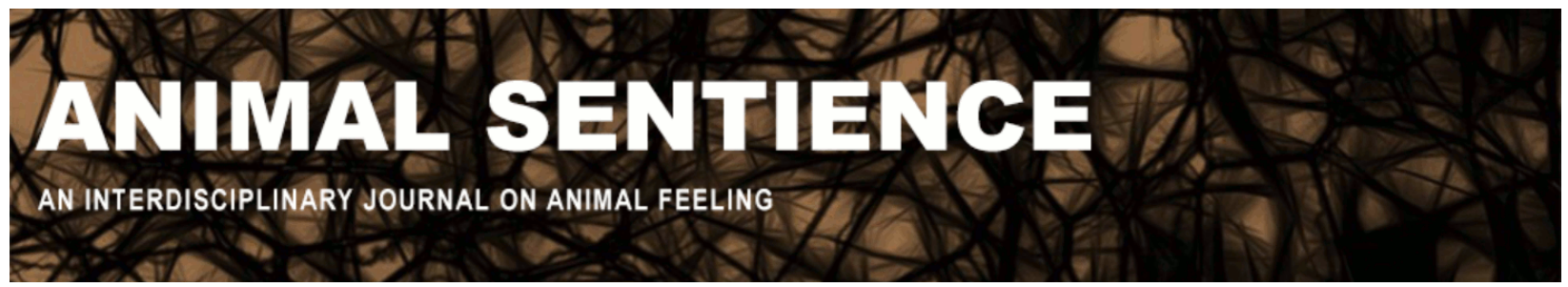

Freire, Rafael and Hazel, Susan J. (2017) Are chicken minds special?. Animal Sentience 17(4)

DOI: $10.51291 / 2377-7478.1223$

Date of submission: $2017-10-12$

Date of acceptance: 2017-10-16 (c) 


\title{
Are chicken minds special?
}

Commentary on Marino on Thinking Chickens

\author{
Rafael Freire \\ School of Animal and Veterinary Sciences \\ Charles Sturt University, Australia \\ Susan J. Hazel \\ School of Animal and Veterinary Sciences \\ University of Adelaide, Australia
}

\begin{abstract}
The number of publications on chicken cognition and emotion exceeds that on most birds and is comparable to the number of publications on some more "advanced" mammals. We argue that the chicken is an excellent model for this type of research because of (1) the presence of well-established fundamental mental processes in the chicken, (2) a challenging ethological environment and (3) social pressures that may have facilitated the evolution of cognitive abilities similar to those of some mammals. Marino's $(2017 a, b)$ review provides an excellent foundation for the continued study of complex mental abilities in this species.
\end{abstract}

Rafael Freire is Senior Lecturer in Animal Behaviour and Welfare and has published on chicken behavior, cognition, welfare and magnetic orientation.

https://science.csu.edu.au/schools/animalvet/staff/profiles/senior-lecturers/raf-freire

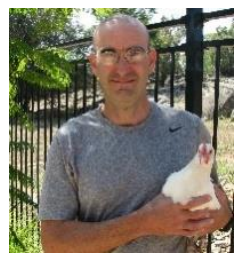

Susan Hazel is Senior Lecturer in Animal Behaviour, Welfare \& Ethics and has published on student attitudes to chickens as well as cognition, behavior and welfare in other species.

https://researchers.adelaide.edu.au/profile/susan.hazel

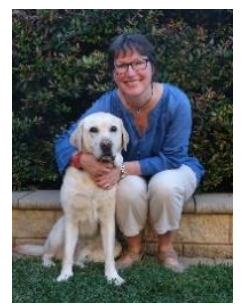

The recent review by Marino $(2017 a, b)$ highlighted the great deal that we know about cognition, emotion and more "complex" levels of mental processing in the chicken. Domestic chickens have been a popular model for the scientific study of development, behaviour, neuroscience and physiology, to name just a few areas, for some time. This may be partly due to their wide availability and ease of rearing and care. For example, advances in brain laterality and function and the neurological basis for aversion learning have been aided by the decussation of the optic nerve and easy precocial rearing (Rogers 1995). In addition, it has been argued that because they are a domesticated species less fearful of humans and do not have to be obtained from the wild, chickens offer a more ethical choice of subject than other birds used in some areas of research 
(Freire 2011). It is clear from reading Marino's review that considerable research has also been undertaken on chicken cognition and emotion. To put this into perspective, a literature search indicates that more papers have been published on "emotion or cognition" in the chicken than in any other bird, except pigeons (Figure 1).

Figure 1: Number of "emotion or cognition" publications from 1967-2017* cited in Web of Science for different species.

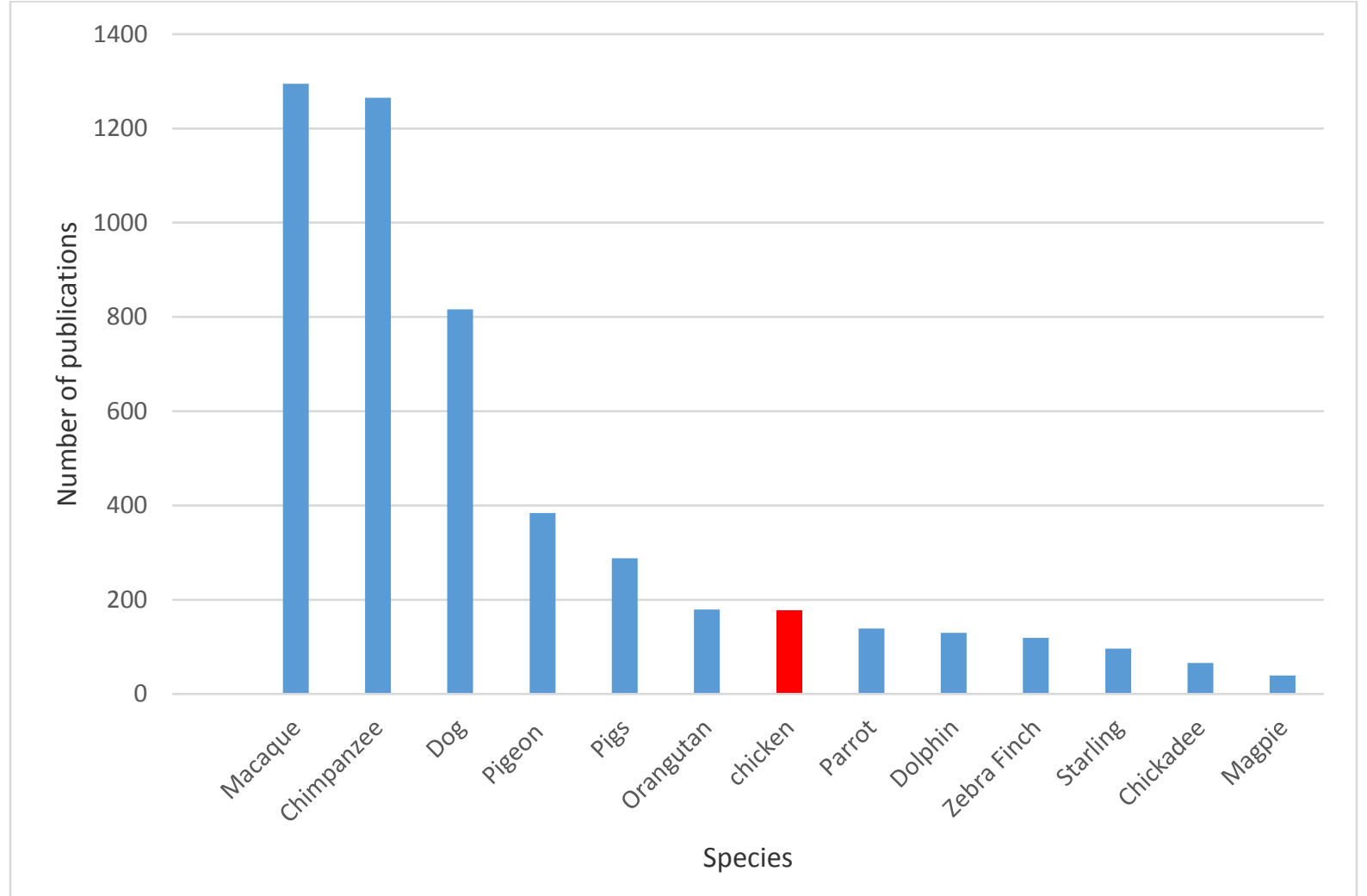

*1967 was chosen as the start year for this search, as this was the oldest paper on animals cited by Marino (2017a). The science and social science citation indexes of Web of Science were used. Common names as above were used, as well as Latin names, to search for publications for each species. Searched 8/10/2017.

The popularity of chickens (relative to other birds) in emotion and cognition research does appear to come as a surprise to some people. As cited by Marino, when college students participate in a practical class to clicker-train chickens, they are more likely to believe not only that chickens are intelligent with individual personalities, but also that they can experience complex emotions (boredom, frustration and happiness) (Hazel et al. 2015). This study has been picked up widely due to interest in the changes in student attitudes; what was not included in the publication is the more fascinating story of how quickly and easily the chickens learn. In the practical class, chickens are taught a simple form of associative learning, operant or instrumental conditioning, using a bridge (the clicker) followed by a food reward. Chickens are shaped using successive approximations to peck on a red target (a small red circle), and once that is accomplished, other colours are added (green, yellow, purple) to demonstrate colour discrimination. Chickens continue to peck on the red target and not on the other colours. The practical class breaks every rule in training 101 when it comes to setting up the environment to reduce distractions and 
maintain consistency. It lasts 2 hours, with a large proportion devoted to students practicing their technical skills without the chickens, as otherwise their reflexes are far too slow to keep up with the speed of the pecks. The room is filled with up to 24 students who work in pairs, and each table has a chicken at each end. Each week a different group of students participates.

The students are astounded at how each chicken perceives "their" clicker, as there are up to 12 clickers being used simultaneously in a relatively small room. There are always some chickens that learn more quickly than others, and some that we don't continue to use, as they appear too stressed by the noise and all of the students, although they will perform well in a quiet room. And yet each year almost all of the chickens learn to peck the red target and discriminate by colour within 1 to 3 weeks. As discussed by Marino, there is believed to be an interplay between fundamental cognitive processes (e.g., associative learning as described above) and higher-level capacities (Shettleworth 2010). This parallels the simple individual behaviours that lead to apparently complex collective behaviours, such as in a school of fish or flocking birds. Thus, chickens' ability to learn associations quickly may be a foundation for building more complex behaviours such as learning multiple social interactions over time.

Having overcome the prejudice of "it's only a chicken," perhaps the next question is whether chickens are actually a good model for emotion and cognition research, or just happen to be a handy subject.

Chickens have a complex ethological life, including an established and carefully maintained pecking order, recently described in detail by Nicol (2015). For some time, some authors have considered that the human collection of complex cognitive abilities has arisen from the pressures of an increasingly complex social environment (e.g., Flinn et al. 2005). Dawkins (1993) first provided a compelling argument as to the possibility of complex cognitive and emotional processes in the chicken. The argument was based, in part, on viewing complex mental processes as adaptations to the challenging social environment in which chickens live. The extent to which these challenges may be similar to those that shaped our own cognitive adaptation is debatable, and difficult to answer, though it is interesting to note that some people do relate closely with chickens. As an example, in Australia, chickens have helped a 10-year-old girl develop her understanding of people and social interactions, an experience which she has developed into a program to help others with Asperger's diagnosis (Marsellos \& Buchanan 2017).

At the time, Dawkins's (1993) argument for complex mental abilities in the chicken bucked the trend to consider consciousness and other complex mental abilities as most likely to be found in big, intelligent animals (i.e., apes, monkeys, cetaceans). No doubt, the pull of comparative anatomy led many researchers to look for these abilities in animals most similar to the ones where we know these abilities exist (i.e., us). Yet, any undergraduate zoologist will tell you that similar anatomical structures can arise due to similar environmental pressures on adaptation; and they can no doubt go on and list the impressive similarities between the cephalopod and mammalian eye. If complex mental abilities have been shaped by evolutionary processes, then it would appear feasible for "human-like" emotions and complex mental abilities to have appeared in other animals under similar (perhaps social) selection pressures. Marino (2002) herself has argued that convergent evolution of cognitive processes may have occurred between cetacean and primate lineages. As presented by Marino (2017a), there are many reasons to consider the chicken an excellent model for understanding complex cognitive and emotional processes at many levels and there are perhaps a few prejudices that we need to overcome to realise their potential 
contribution to science. Chicken minds aren't more special than the minds of any other species; they just show fascinating adaptation to a complex ethological and social environment. Rather as ours do.

\section{References}

Dawkins, M. S. (1993). Through our eyes only?. WH Freeman.

Flinn, M. V., Geary, D. C., \& Ward, C. V. (2005). Ecological dominance, social competition, and coalitionary arms races: Why humans evolved extraordinary intelligence. Evolution and Human Behavior, 26(1), 10-46.

Freire, R. (2011). Ethical advantages of using domestic bird species for magnetic orientation research. Communicative \& Integrative Biology, 4(1), 84-85.

Hazel, S. J., O'Dwyer, L., \& Ryan, T. (2015). "Chickens are a lot smarter than I originally thought": Changes in student attitudes to chickens following a chicken training class. Animals, 5(3), 821-837.

Marino, L. (2002). Convergence of complex cognitive abilities in cetaceans and primates. Brain, Behavior and Evolution, 59(1-2), 21-32.

Marino, L. (2017a). Thinking chickens: A review of cognition, emotion, and behavior in the domestic chicken. Animal Cognition, 20(2), 127-141.

Marino, L. (2017b). The inconvenient truth about thinking chickens. Animal Sentience 17(1).

Marsellos, B., \& Buchanan, K. (2017, 25 September). Chicken therapy program for people with Asperger's developed by 10 yo girl.

Nicol, C. J. (2015). The behavioural biology of chickens. CABI.

Rogers, L. J. (1995). The development of brain and behaviour in the chicken. CAB International. Shettleworth, S. J. (2010). Cognition, evolution, and behavior. Oxford University Press. 